\title{
A survey of risk assessment skills and training among health professionals in Pakistan
}

\author{
Rameez Zafar ${ }^{1}$ MRCPsych, Khurram T. Sadiq ${ }^{2}$ MBBS and Sylvia A. Khan ${ }^{3}$ MBBS
}

${ }^{1}$ Consultant Adult Psychiatrist, Peter Hodgkinson Centre, Lincoln, UK, email zafarrameez@hotmail.com ${ }^{2} \mathrm{SHO}$, Forensic Psychiatry, Francis Willis Unit, Lincoln, UK ${ }^{3} \mathrm{SHO}$, Manthorpe Centre, Grantham, UK

0 ver the past two decades, psychiatric services have evolved globally and generally there has been a gradual transition from hospital-based practice to a more community-based approach. The stigma associated with psychiatry has somehow diminished and society in general can now relate better to this field of medicine.

Risk assessment is an integral part of mental healthcare, and mental health professionals should be well versed and trained in it, but this is not always the case. There are large differences in the way mental health professionals from the higher-income and lower-income countries perceive psychiatry, particularly risk assessment. Training in the recognition and management of suicidal risk is of crucial importance for the prevention of suicidal behaviour. This perception formed the basis of the survey reported in this paper, the purpose of which was to obtain a comprehensive picture of risk assessment conducted with patients presenting with self-harm to health professionals in Peshawar, Pakistan.

Peshawar is the capital of North West Frontier Province (NWFP), one of the four provinces of Pakistan. Although rich in culture, it is deprived in relation to health services.

Psychiatry is still not appreciated as a healthcare service in Pakistan, and an attempt to commit suicide is still considered a crime, to be reported to the police, even before any medical help is sought (Khan, 1998). Most people who have harmed themselves are admitted to general medical wards because they need physical intervention and are not usually referred to psychiatry owing to the stigma attached (Karim et al, 2004).

The aim of this study was to explore the attitudes of trainees from a range of clinical backgrounds towards self-harm and their clinical experience in dealing with the problem. It looked at the risk assessment skills and training provided to trainees working in different specialties in two different hospitals in Peshawar, Pakistan. It also explored the aetiology, manner and subsequent management of self-harm among patients.

\section{Methods}

A questionnaire was designed and distributed among trainees in internal medicine, general surgery and accident and emergency medicine, as well as a few psychiatric trainees (see Table 1). They all worked in Khyber Teaching Hospital or
Lady Reading Hospital, Peshawar, Pakistan. Fifty-six of the 80 questionnaires distributed were returned. The questionnaire dealt with the training which the trainees had received in risk assessment, the use of guidelines and protocols, and their understanding of the motives and the methods used for selfharm. It also examined the subsequent management as well as follow-up procedures.

\section{Results}

The respondents were 39 men and 17 women, with an age range of $25-45$ years (Table 1 ). Fifty-one (91\%) of the trainees had regularly seen patients presenting after self-harm at some stage of their routine practice. None of the trainees, including the psychiatric trainees, had received any formal training in risk assessment; however, 15 (27\%) of them had seen some sort of guidelines or protocols for risk assessment.

Despite the fact that the police have to be involved in every case of self-harm, the majority of the trainees $(31 ; 55 \%)$ thought that the medical staff should be the first contact for these individuals (Table 2).

Three-quarters of the trainees thought that medication overdose was the most common method of self-harm (Table 2 ). According to the trainees surveyed, the most common age group presenting with self-harm was 20-30 years. Thirtyfour $(61 \%)$ of the trainees reported that the majority of those who self-harmed were female, while $9 \%$ of them suggested

Table 1 Attributes of the survey respondents

$\begin{array}{lrr} & n & (\%) \\ \text { Age (years) } & & \\ 25-35 & 51 & (91) \\ 36-45 & 5 & (9) \\ \text { Gender } & & \\ \text { Female } & 17 & (30) \\ \text { Male } & 39 & (70) \\ \text { Specialties } & & \\ \text { Medicine } & 44 & (78) \\ \text { Psychiatry } & 6 & (11) \\ \text { Accident and emergency } & 5 & (9) \\ \text { Surgery } & 1 & (2) \\ \text { Training } & & \\ \text { Formal training } & 0 & (0) \\ \text { Awareness of guidelines } & 15 & (27)\end{array}$


Table 2 Perception of respondents of the nature of self-harm

$\begin{array}{lrr} & n & (\%) \\ \text { Gender more commonly involved in self-harm } & & \\ \text { Male } & 17 & (30) \\ \text { Female } & 34 & (61) \\ \text { Similar } & 5 & (9) \\ \text { Best first port of contact } & & \\ \text { Medical staff } & 31 & (55) \\ \text { Relatives } & 22 & (39) \\ \text { Police } & 3 & (5) \\ \text { Common methods of self-harm } & & \\ \text { Overdose on medication } & 42 & (75) \\ \text { Overdose on illicit drugs } & 8 & (14) \\ \text { Laceration/cutting } & 3 & (5) \\ \text { Other } & 3 & (5) \\ \text { Reasons for self-harm } & & \\ \text { Social constraints } & 31 & (55) \\ \text { Life events } & 11 & (19) \\ \text { Frustration } & 6 & (11) \\ \text { Mental health problems } & 6 & (11) \\ \text { Impulsivity } & 1 & (2) \\ \text { No response } & 1 & (2) \\ \text { Choice of treatment } & & \\ \text { Combined pharmacotherapy and psychological } & 30 & (53) \\ \text { therapy } & & \\ \text { Pharmacotherapy } & 24 & (43) \\ \text { Psychological treatment } & 2 & (4) \\ \text { Further follow-up } & & \\ \text { Necessary } & 16 & (29) \\ \text { Not necessary } & 40 & (71) \\ & & \end{array}$

that the ratio of self-harm behaviours between males and females was about equal (Table 2).

A majority of the trainees felt confident in doing some form of self-harm assessment, while the others felt either embarrassed or anxious about the whole issue.

Thirty-one (55\%) of the trainees believed that self-harm was mainly the result of social constraints. Another 19\% attributed it to life events. Interestingly, only $11 \%$ thought that it was due to mental health problems (Table 2).

A majority of the trainees believed that pharmacotherapy alone or in combination with psychological therapy was the preferred means of management, while only two (4\%) favoured psychological treatment alone.

Because of lack of awareness, more than $70 \%$ of the trainees did not think that a follow-up appointment was necessary; however, the remaining trainees felt that some community or out-patient follow-up should be considered for these individuals (Table 2).

\section{Discussion}

There were no major differences between the psychiatric and non-psychiatric trainees in terms of their training in risk assessment when dealing with self-harm patients. Only $11 \%$ of the trainees considered self-harm to be a result of mental health problems. The fact that self-harm is reported to the police indicates that it is treated as some sort of crime rather than being considered a mental health issue (Khan, 1998). However, trainees are aware that assessment is still warranted.

Apart from a few exceptions there is no well-defined training system in general psychiatry in Pakistan (Farooq, 2001). Therefore, the current scenario is far from ideal. This prevented us from comparing a group of psychiatric trainees with a group of non-psychiatric trainees. Nevertheless, further research could be conducted to examine whether this study reflects the general pattern across most hospitals, in different cities in Pakistan.

The sample in our study was fairly small, but taking into account the fact that the concept of proper risk assessment was new to most of them, the response rate was still good. It would have been useful to include more psychiatric trainees but the study was limited to two hospitals with relatively small psychiatric wings.

The study was based on the self-reported accounts of the trainees working in different departments and the records of the patients were not checked. Therefore the actual proportions of self-harm methods and age groups were not known. However, the general impression and views of the trainees did give us useful insight into this area.

This study was conducted in a relatively deprived part of the country, where resources are somewhat limited; therefore the conclusions cannot readily be generalised to other areas of the country.

In Pakistan, mental ill-health is still strongly stigmatised (Karim et al, 2004). Individuals presenting after an episode of self-harm are dealt with in general wards and they are reluctant to be referred to psychiatric services. In fact, more than $50 \%$ of the trainees surveyed felt that if self-harm was associated with mental illness, this would lead to a greater stigmatisation of the patients.

Pakistani society in general is very reactive and sensitive about the social implications of any suicidal behaviour. Patients are often accompanied by their friends and family members and word of mouth spreads rapidly. Keeping information confidential about this client group is sometimes not possible.

Forty-four (78\%) of the trainees in this study were from general medicine and only six (11\%) were from psychiatry, which strongly indicates that non-psychiatric trainees play a key role in assessing patients who present after an episode of self-harm. Women were reported to form the majority of the patients ( $61 \%$ of trainees believed this to be the case), with the common trigger being their social circumstances (Haider \& Haider, 2001). This may have some cultural implications in an orthodox society where attitudes to self-harm are in any case quite negative (Khan et al, 1996). The care of female patients by female clinicians may be considered appropriate.

As psychiatrists are not involved in most cases of self-harm, non-psychiatric trainees need to be better trained in clinical risk assessment. Some trainees did recognise that there were deficits in practice which impaired the quality of care. It was suggested that supervision and further training through workshops, seminars and conferences, as well as enhanced policies and procedures for dealing with self-harm, were urgently required to reduce the risk of stigmatisation within the medical establishment and wider society. To this end, care pathways for the management of this client group and guidelines for ensuring confidentiality would help a great deal.

Keeping in view the lack of proper training and exposure to this aspect of medicine, the trainees in this study still showed at least some understanding of proper risk assessment. There is clearly a need to do further studies in this field, especially in the major teaching hospitals in the country, to ascertain the current trends and practices in self-harm assessments, as well as attitudes of doctors towards psychiatric 
disorders in general (Farooq et al, 2005). Studies of this kind should promote better awareness and understanding among trainees of the importance of carrying out proper risk assessment and management. Substantial resources also need to be invested in order to restructure the whole system.

\section{References}

Farooq, S. (2001) Psychiatric training in developing countries. British Journal of Psychiatry, 179, 464.
Farooq, S., Akhtar, J., Anwar, E., et al (2005) The attitude and perception of hospital doctors about the management of psychiatric disorders. Journal of the College of Physicians and Surgeons Pakistan, 15, 552-555.

Haider, I. S. \& Haider, I. (2001) Deliberate self harm. Pakistan Journal of Medical Sciences, 17, 151-155.

Karim, S., Saeed, K., Rana, M. H., et al (2004) Pakistan mental health country profile. International Review of Psychiatry, 16, 83-92.

Khan, M. M. (1998) Suicide and attempted suicide in Pakistan. Crisis, 19, 172-176.

Khan, M. M., Islam, S. \& Kundi, A. K. (1996) Parasuicide in Pakistan: experience at a university hospital. Acta Psychiatrica Scandinavica, 93, 264-267.

\title{
The psychological and psychosocial impact of the Pakistan Kashmir earthquake after 8 months: a preliminary evaluation by PACTT
}

\author{
PACTT: Pakistan-Aberdeen Collaborative Trauma Team*
}

$\mathrm{N}$ atural disasters are complex events that challenge the coping abilities of individuals and communities (Alexander, 2005). They are characterised by substantial loss, physical injury and economic hardship, as well as by extensive internal displacement and damage to the infrastructure, as exemplified by the Pakistan Kashmir earthquake of 8 October 2005. Measuring 7.6 on the Richter scale, it affected an area of 30000 square miles and a population of 3.6 million. Approximately 90000 were killed, 200000 were injured and 3.5 million were left homeless (Khan, 2006). Based on a literature review and estimates from the World Health Organization (WHO), the National Plan of Action for Mental Health and Psychosocial Relief of Earthquake Survivors anticipated high levels of trauma-related psychopathology (Rana et al, 2006).

A disaster of such magnitude poses enormous problems for mental health and psychosocial care (Ghodse \& Galea, 2006), particularly in the context of high levels of untreated pre-earthquake morbidity in Pakistan (Mirza \& Jenkins, 2004) plus the estimated post-earthquake morbidity (Rana et al, 2006). The absence of evidence-based 'best practice' for culturally sensitive psychological interventions in relation to such a disaster led to a miscellany of unintegrated interventions being implemented in the earthquake-affected region (Khan, 2006). The blanket provision of such interventions is not a cost-effective or efficient strategy and may even be harmful (Alexander, 2005).

Fundamental to informing a culturally sensitive disaster response strategy is an epidemiological and needs assessment approach (van Ommeren et al, 2005), but post-disaster ethical, methodological and logistic factors may impede the use of representative sampling methods and culturally validated instruments (Klein \& Alexander, 2007).

To test the feasibility of undertaking a culturally informed, epidemiological, community-based survey of the traumatic effect of the earthquake, the Pakistan-Aberdeen Collaborative Trauma Team (PACTT) conducted a pilot study in the earthquake-affected region of Azad Jammu and Kashmir (AJ\&K). A key objective of the pilot was to test the acceptability of the assessment instruments in terms of their cultural and linguistic sensitivity (e.g. with regard to symptom expression), length, comprehensibility and process of administration. Assessment instruments comprised a trauma-modified version of the Composite International Diagnostic Interview (CIDI-TM) developed by PACTT and the Screening Instrument for Traumatic Stress in Earthquake Survivors (SITSES; Basoglu et al, 2001). This paper reports on the outcome of that evaluation and presents preliminary findings derived from the SITSES in relation to the psychological and psychosocial impact of the Pakistan Kashmir disaster after 8 months.

*PACTT consists of the following members: Pakistan - Brigadier Mowadat H. Rana, ${ }^{1}$ Dr Sohail Ali, ${ }^{2}$ Dr Babur Yusufi ${ }^{3}$;

Aberdeen, Scotland, UK - Professor David A. Alexander, ${ }^{4}$ Dr Susan Klein, ${ }^{5}$ Professor Amanda J. Lee, ${ }^{6}$ Dr Gareth T. Jones, ${ }^{7}$ Professor Gary Macfarlane ${ }^{8}$ ${ }^{1}$ Advisor in Psychiatry, Military Hospital Rawalpindi; ${ }^{2}$ Consultant Psychiatrist, Military Hospital Rawalpindi; ${ }^{3}$ Specialist Registrar, Community Learning Disability Service, Mile End Hospital, London; ${ }^{4}$ Director, Aberdeen Centre for Trauma Research, The Robert Gordon University, Aberdeen;

${ }^{5}$ Reader, Aberdeen Centre for Trauma Research, The Robert Gordon University, Aberdeen; ${ }^{6}$ Professor of Medical Statistics, Department of General Practice and Primary Care, University of Aberdeen; ${ }^{3}$ Senior Lecturer in Epidemiology, Epidemiology Group, Department of Public Health, School of Medicine, University of Aberdeen; 'Professor of Epidemiology, Epidemiology Group, Department of Public Health, School of Medicine, University of Aberdeen Corresponding author: Dr S. Klein, Reader, Aberdeen Centre for Trauma Research, Faculty of Health and Social Care, The Robert Gordon University, Scotland, UK, email s.klein@rgu.ac.uk 